\title{
Meta-analysis on the use of hyaluronic acid gel to prevent intrauterine adhesion after intrauterine operations
}

\author{
FEI ZHENG ${ }^{1 *}$, XIN XIN $^{2 *}$, FEI HE $^{1}$, JIANYONG LIU $^{1}$ and YUECHONG CUI ${ }^{3}$ \\ ${ }^{1}$ Department of Obstetrics and Gynecology, Yiwu Maternity and Children Hospital, Jinhua, Zhejiang 322000; \\ ${ }^{2}$ Department of Obstetrics and Gynecology, Benxi Central Hospital, Benxi, Liaoning 117000; \\ ${ }^{3}$ Department of Human Health and Human Services, Yiwu Maternity and Children Hospital, \\ Jinhua, Zhejiang 322000, P.R. China
}

Received May 29, 2019; Accepted January 6, 2020

DOI: $10.3892 /$ etm.2020.8483

\begin{abstract}
Intrauterine adhesion (IUA) is a severe complication that occurs following abortion, and hyaluronic acid gel has been used to prevent IUA following intrauterine operation. The present study aimed to systematically evaluate the efficacy of hyaluronic acid gel in preventing IUA following intrauterine operation. In the current study, a literature search was performed using PubMed, Cochrane Library and EMbase databases for randomized controlled clinical trials that used hyaluronic acid gel as an adjuvant therapy following intrauterine operation. The terms 'hyaluronic acid', 'intrauterine adhesions', 'Asherman's syndrome', 'IUA', 'dilatation and curettage', 'abortion' and 'hysteroscopic' were used to search for articles published online before July 31, 2018. RevMan 5.3 software was used to analyze the indicators of uterine cavity adhesion formation and pregnancy rates following intrauterine operation. A total of seven randomized controlled clinical studies were included, consisting of 952 patients who underwent intrauterine operation. The meta-analysis indicated that the use of hyaluronic acid gel reduced the incidence of IUA [relative risk $(\mathrm{RR})=0.42 ; 95 \%$ confidence interval $(\mathrm{CI})=0.30-0.57 ; \mathrm{P}<0.001]$ and the score for IUA after an intrauterine operation (mean difference $=-1.29 ; 95 \%=-1.73$ to $-0.84 ; \mathrm{P}<0.001)$. A subgroup analysis revealed that the preventive effect of hyaluronic acid gel on IUA was not affected by the type of intrauterine operation, namely abortion $(\mathrm{RR}=0.40$; $95 \% \mathrm{CI}=0.26-0.62 ; \mathrm{P}<0.001)$ and hysteroscopy $(\mathrm{RR}=0.44 ; 95 \%$ $\mathrm{CI}=0.28-0.68 ; \mathrm{P}<0.001)$. The preventive effect of hyaluronic acid gel on IUA was also not affected by primary disorders/diseases including the following: Abortion $(\mathrm{RR}=0.48$; $95 \% \mathrm{CI}=0.29-0.78$;
\end{abstract}

Correspondence to: Dr Yuechong Cui, Department of Human Health and Human Services, Yiwu Maternity and Children Hospital, 320 South Gate Street, Yiwu, Jinhua, Zhejiang 322000, P.R. China E-mail: 1026984758@qq.com

*Contributed equally

Key words: hyaluronic acid gel, intrauterine adhesions, intrauterine operation, pregnancy
$\mathrm{P}=0.003)$; IUA ( $\mathrm{RR}=0.38 ; 95 \% \mathrm{CI}=0.21-0.67 ; \mathrm{P}<0.001)$ and submucosal myoma of the uterus, endometrial polyps or mediastinum uterus $(\mathrm{RR}=0.40 ; 95 \% \mathrm{CI}=0.18-0.90 ; \mathrm{P}=0.03)$. Hyaluronic acid gel improved pregnancy rates after intrauterine operations $(\mathrm{RR}=1.94 ; 95 \% \mathrm{CI}=1.46-2.60$; $\mathrm{P}<0.001)$. In conclusion, hyaluronic acid gel was indicated to significantly reduce the incidence of IUA following intrauterine operation, regardless of the type of intrauterine operation or the presence of primary diseases. Treatment with hyaluronic acid gel was also revealed to increase pregnancy rates following intrauterine operation.

\section{Introduction}

Intrauterine adhesions (IUA), also known as Asherman's syndrome, were first described in 1948 by Joseph G. Asherman (1). The causes of IUA include dilatation/curettage, postpartum hemorrhage, hysteromyomectomy, hysteroscopic operation and genital tuberculosis (2-4). The main clinical manifestations of IUAs are hypomenorrhea, recurrent abortion, amenorrhea, periodic hypogastralgia, infertility and altered placental implantation, which are conditions that can cause harm to patient health (5). Currently, the treatment for IUA is hysteroscopic adhesiolysis; however, there is still a high recurrence rate after this treatment method (6). A previous study reported re-adhesion rates of up to $62.5 \%$ after hysteroscopic adhesiolysis (6). Therefore, the prevention of IUA after intrauterine operations is particularly important.

Hyaluronic acid is a highly polymerized chain of disaccharides and a mucopolysaccharide that has important physiological functions in the body, including lubrication of the joints, adjusting the permeability of blood vessel walls, regulating the spread and transport of proteins and electrolytes and promoting wound healing (7). Hyaluronic acid can bind with a large number of water molecules and improve tissue hydration, enhance cell resistance to mechanical injury and reduce post-traumatic granulation tissue and fibrous tissue formation $(8,9)$. Due to its unique biocompatibility and enzymatic biodegradation, hyaluronic acid is often used to prevent postoperative tissue adhesion $(8,9)$. At present, hyaluronic acid gel is widely used after intrauterine operation to prevent IUAs, and this therapy has achieved some beneficial effects (10); however, the rates of adhesion and pregnancy after an intrauterine operation 
are still unclear. The current paper reports a meta-analysis on the existing literature of clinical randomized controlled trials on the use of hyaluronic acid gel following intrauterine operations.

\section{Materials and methods}

Literature search. The Cochrane Library (https://www. cochranelibrary.com/), Embase (https://www.embase.com/) and PubMed (https://www.ncbi.nlm.nih.gov/pubmed/) databases were searched for articles published before July 312018 , using the terms 'hyaluronic acid', 'intrauterine adhesions', 'Asherman's syndrome', 'IUA', 'dilatation and curettage', 'abortion' and 'hysteroscopic'. No language limit was set on the original publication.

Study inclusion and exclusion criteria. The present study included randomized controlled clinical studies that used hyaluronic acid gel following an intrauterine operation. The subjects were selected if the women were considered to be of reproductive age (18-40 years), had undergone an intrauterine operation and if there were at least two treatment groups in the trial (with or without the use of hyaluronic acid gel) regardless of other assistive interventions, including the administration of estrogen or the use of oral contraceptives.

Studies were excluded if they were case reports, articles without specific data or studies with clear defects in experimental design.

Evaluation index. The efficacy of hyaluronic acid gel interventions was evaluated using two outcome measures. The first was the incidence rate of IUAs, according to the grading method as described by the American Fertility Society in 1988 (11) and the second was pregnancy rate. The outcome measures were assessed according to the following equation: Number of cases (IUA of pregnancy)/total number of cases.

Study quality evaluation and statistical analysis. The literature screening, bias risk assessment and data extraction were conducted systematically by two independent evaluators. Risk assessments were performed using the clinical randomized controlled trial evaluation method as described by the Cochrane reviewer's handbook version 5.1 (12). All data were analyzed using the RevMan 5.3 software (Cochrane).

The statistical heterogeneity of each study result was analyzed using the $\chi^{2}$ test and the statistical significance was set at a cut-off value of $\mathrm{P}=0.1 . \mathrm{P}<0.01$ indicated statistical heterogeneity of the study. $\mathrm{I}^{2}$ was used to evaluate the heterogeneity of the results. An $\mathrm{I}^{2}$ score of $<25 \%$ suggested that the heterogeneity may not be important; however, it indicated significant heterogeneity when it was $>50 \%$. If the $\mathrm{I}^{2}$ was $>75 \%$, a high degree of heterogeneity was indicated. When the $\mathrm{I}^{2}$ was $>50 \%$, a random-effects model was adopted followed by a subgroup and sensitivity analyses. When the $\mathrm{I}^{2}$ was $<50 \%$, a fixed-effects model was adopted.

All subjects were included in the analysis and were divided into the treatment group, which received hyaluronic acid gel treatment after an intrauterine operation, or the control group, which did not receive treatment with hyaluronic acid gel after intrauterine operation. A dichotomous variable was expressed as the relative risk (RR) and 95\% confidence interval (CI), while a continuous variable was expressed as the mean difference (MD).

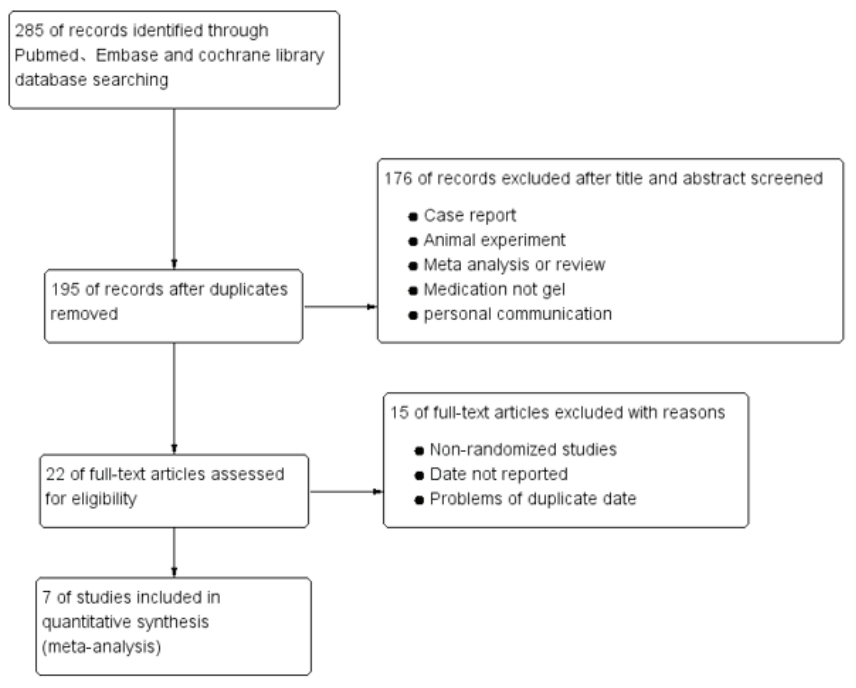

Figure 1. Flowchart depicting the method behind the selection of the studies.

\section{Results}

Baseline characteristics. A total of 285 studies were initially selected. A total of seven were included in the final analysis after screening (Fig. 1) (10,13-18). All 7 studies compared the efficacies of using hyaluronic acid gel as an adjuvant therapy (treatment group) against a control group (without the use of hyaluronic acid gel) after an intrauterine operation. A total of 952 women of reproductive age who underwent intrauterine operations were included. From these patients, 455 had been treated with hyaluronic acid gel postoperatively and 497 were controls. Among the 7 studies, 3 used common abortive procedures such as intrauterine operations $(14,15,17)$ and 4 were performed using hysteroscopy $(10,13,16,18)$. A total of six studies were performed using direct injection with the gel $(10,16,18)$ and one used a temporary adhesion barrier, which hydrated into a gel within 24-48 h of administration into the patient (17). There were no statistically significant differences in baseline parameters between the treatment and control groups, as presented in Table I (10,13-18). The results of the quality assessment of the included studies are presented in Fig. 2. Allocation concealment methods for all the studies were not included.

Meta-analysis results. All seven studies reported the incidence rate of IUAs; however, only 4 reported the IUA score after the intrauterine operation (14-16,18). As indicated in Figs. 3 and 4, the hyaluronic acid gel reduced the incidence of IUA after an intrauterine operation $(\mathrm{RR}=0.42 ; 95 \% \mathrm{CI}=0.30-0.57 ; \mathrm{P}<0.001)$ and the IUA score after an intrauterine operation $(\mathrm{MD}=-1.29$, $95 \% \mathrm{CI}=-1.73$ to $-0.84, \mathrm{P}<0.001)$. A subgroup analysis revealed that the preventive effect of hyaluronic acid gel on IUAs was not affected by the type of intrauterine operation, as presented in Fig. 5. Abortions had an RR of 0.40 with a $95 \%$ CI of 0.26-0.62 $(\mathrm{P}<0.001)$ and hysteroscopy had an RR of 0.44 with a $95 \%$ CI of $0.28-0.68(\mathrm{P}<0.001)$. The analysis also indicated that the preventive effect of hyaluronic acid gel on IUA was not significantly affected by primary diseases, namely abortion $(\mathrm{RR}=0.48 ; 95 \% \mathrm{CI}=0.29-0.78 ; \mathrm{P}=0.003)$, IUAs $(\mathrm{RR}=0.38$; $95 \% \mathrm{CI}=0.21-0.67 ; \mathrm{P}<0.001)$, and submucosal myoma of uterus 


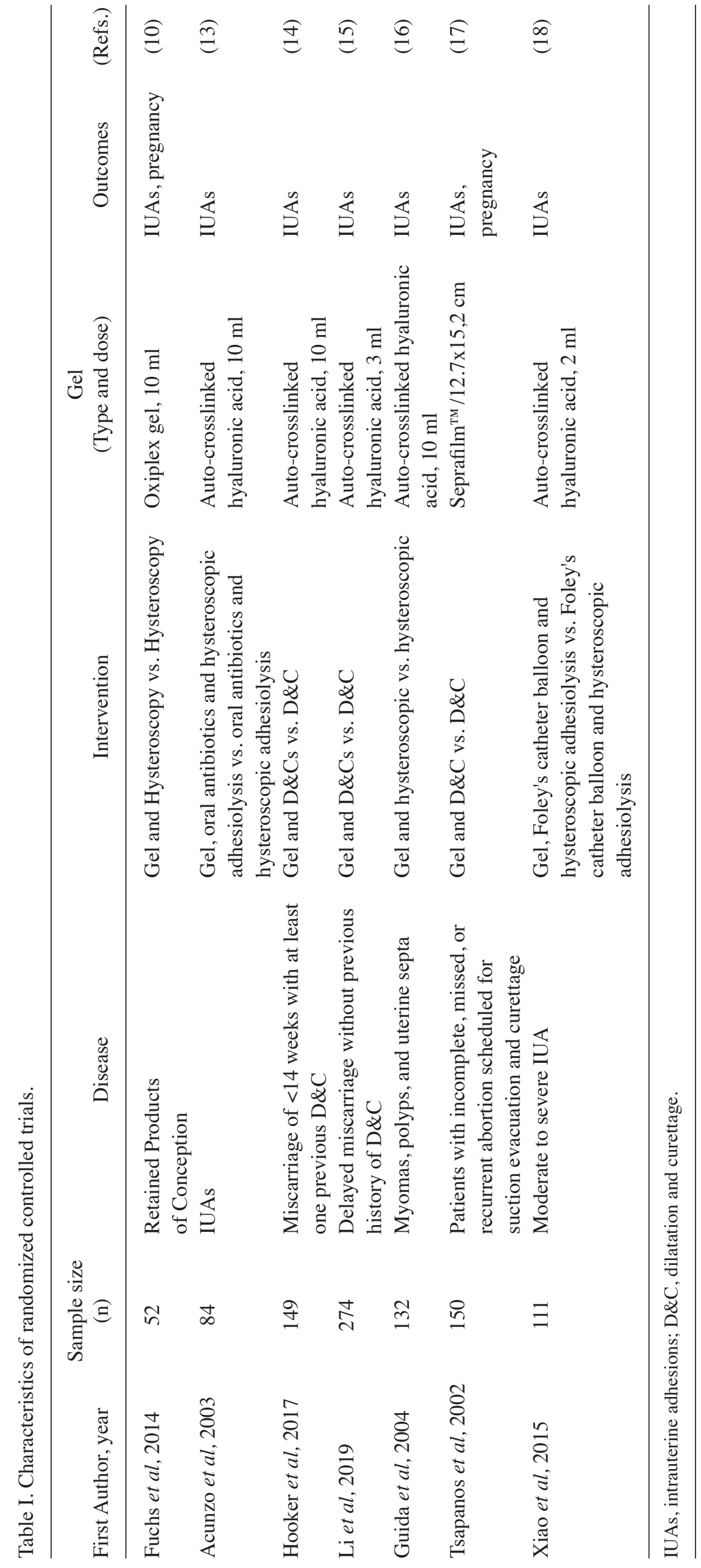




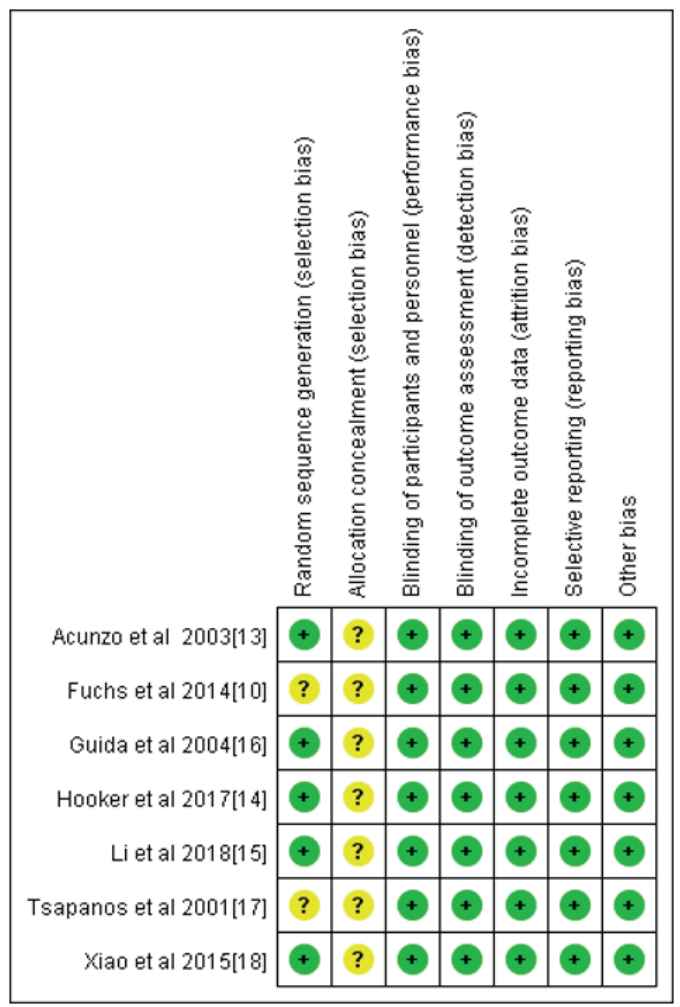

Figure 2. Biased risk assessment chart of studies included in the meta-analysis $(n=7)$. Green indicates a low risk of bias, yellow indicates an unclear risk of bias and red indicates a high risk of bias.

or endometrial polyps or mediastinum uterus $(\mathrm{RR}=0.40 ; 95 \%$ $\mathrm{CI}=0.18-0.90 ; \mathrm{P}=0.03)$, as presented in Fig. 6 . A total of two studies $(10,17)$ reported the effect of the treatment on pregnancy rates and the results demonstrated that the hyaluronic acid gel could improve pregnancy rates after intrauterine operations $(\mathrm{RR}=1.94 ; 95 \% \mathrm{CI}=1.46-2.6 ; \mathrm{P}<0.001)$, as presented in Fig. 7.

\section{Discussion}

A total of seven studies were included in the present meta-analysis; all reported the incidence of IUA after intrauterine operation $(10,13-18)$, but only four reported the IUA score $(14-16,18)$. The results of the current study demonstrated that hyaluronic acid gel reduced IUA incidence and its score following an intrauterine operation. This reduction was not affected by the type of intrauterine operation or the presence of primary diseases. The effect of treatment on pregnancy rates was reported in two studies, and the results indicated that hyaluronic acid gel could improve pregnancy rates following an intrauterine operation $(10,17)$. However, neither of these studies reported additional details such as the use of contraceptives or the number of live births after treatment. These aforementioned studies also did not provide sufficient follow-up data. In the present study, it was not possible to evaluate the effect of hyaluronic acid gel on postoperative pregnancy and live birth rates. None of the seven studies reported gel-related or surgical-related complications, including hemorrhage, perforation or cervical laceration.

Hyaluronic acid is a highly polymerized chain of disaccharides and is a mucopolysaccharide. Due to its irregular coiled state, physical and chemical properties (19), it has important physiological functions in the body, such as lubricating the joints, adjusting the permeability of blood vessel walls, regulating the spread and transport of proteins and electrolytes and promoting wound healing (7). Due to its unique biocompatibility and enzymatic biodegradation, hyaluronic acid is often used to prevent postoperative tissue adhesion $(8,9)$. However, its limited half-life causes it to degrade easily in the body, limiting its effectiveness (20). Hyaluronic acid has also been combined with other biopolymers, such as proteins or peptides, through esterification, cross-linking and other chemical modifications of hyaluronic acids (20). This has led to the production of related compounds, such as hyaluronic acid-carboxymethylcellulose gels, which are widely used to prevent tissue adhesion after surgery (20). In recent years, the preventive effect of hyaluronic acid gel on IUA has attracted wide attention. Hooker et al (14) randomly assigned 152 women to the auxiliary auto-crosslinked hyaluronic acid gel group (intervention group) and the non-assisted auto-crosslinked hyaluronic acid gel group (control group) following dilatation and curettage (D\&C). A follow-up diagnostic hysteroscopy was conducted 8 to 12 weeks after the $\mathrm{D} \& \mathrm{C}$; the results showed that the application of gel after D\&C significantly reduced the incidence of postoperative IUAs. A further study using animal models supported these findings. Huberlant et al (21) performed bilateral uterine curettage in 20 New Zealand white rabbits where one uterine tube in each rabbit was its own control. The rabbits were randomized into two groups where the second uterine tube was treated with either hyaluronic acid or isotonic saline. The results suggested that the use of hyaluronic acid gel in animal models improved fertility and reduced the incidence of IUA.

Previous studies have indicated that hormones, intrauterine balloons and intrauterine devices (IUD), achieved some curative effect in preventing IUAs, but there were no clear indicators as to the improvement of clinical symptoms and pregnancy rates of the patients (22-26). Abu Rafea et al (27) demonstrated that postoperative intrauterine placement of a Foley's catheter balloon exhibited no significant effect on childbirth and pregnancy (27). However, Gupta et al (28) revealed that a postoperative Foley's catheter balloon placement seemed to prevent IUA; however, this is yet to be validated in a study with a larger cohort. Although this method has had some success, the secondary tissue damage and secondary uplink infection caused by a Foley's balloon extraction remains to be determined. Krajčovičová et al (29) indicated that following hysteroscopic adhesiolysis, the probability of using a hyaluronic acid gel and an IUD to restore a normal menstrual cycle was 60.0 and $55.5 \%$ respectively, compared with the probability of the control group of $36.3 \%$. The pregnancy rate of patients treated with the hyaluronic acid gel was $77.7 \%$, significantly higher compared with patients treated with an IUD (60.0\%). It can be inferred that the application of the hyaluronic acid gel is better than an IUD or balloon at improving the pregnancy rate after IUA separation. It was also demonstrated that hyaluronic acid can regulate inflammatory factors, promote the normal epithelialization and regeneration of wound tissues, effectively isolate adjacent healing tissues and prevent the formation of adhesions (30).

In conclusion, the current study indicated that hyaluronic acid gels can significantly reduce IUA incidences after an intrauterine operation. Their efficacy does not appear to be affected 


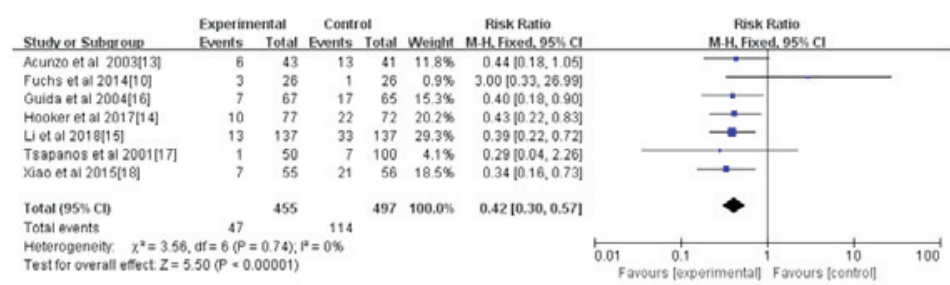

Figure 3. Forest plot of the incidence of intrauterine adhesions after intrauterine operation. CI, confidence interval; df, degrees of freedom; M-H, Mantel-Haenszel.

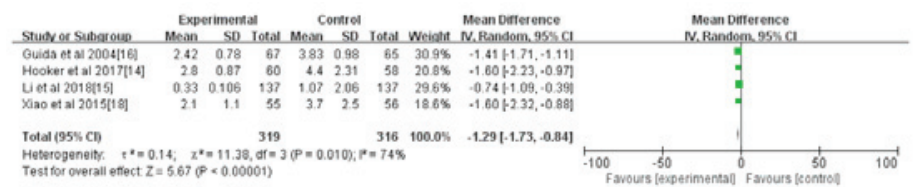

Figure 4. Forest plot of the intrauterine adhesion score after an intrauterine operation. CI, confidence interval; df, degrees of freedom; IV, inverse variance.

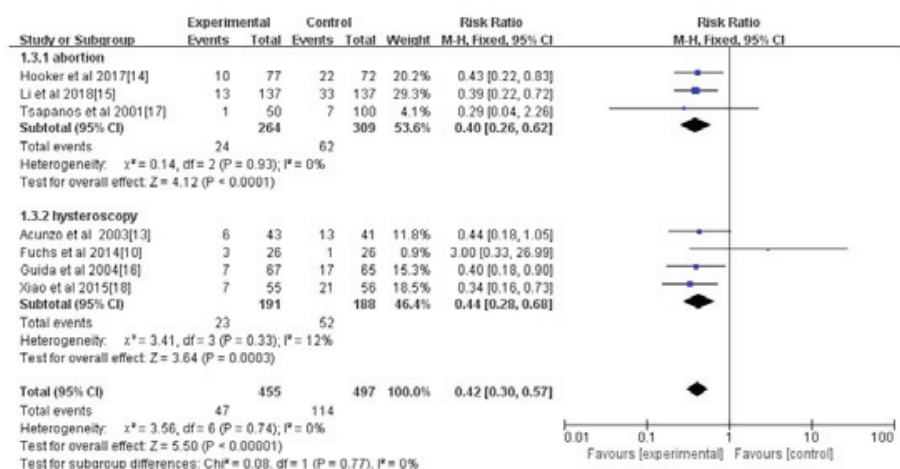

Figure 5. Forest plot of the incidence of intrauterine adhesions between abortion and hysteroscopy after an intrauterine operation. CI, confidence interval; df, degrees of freedom; M-H, Mantel-Haenszel.

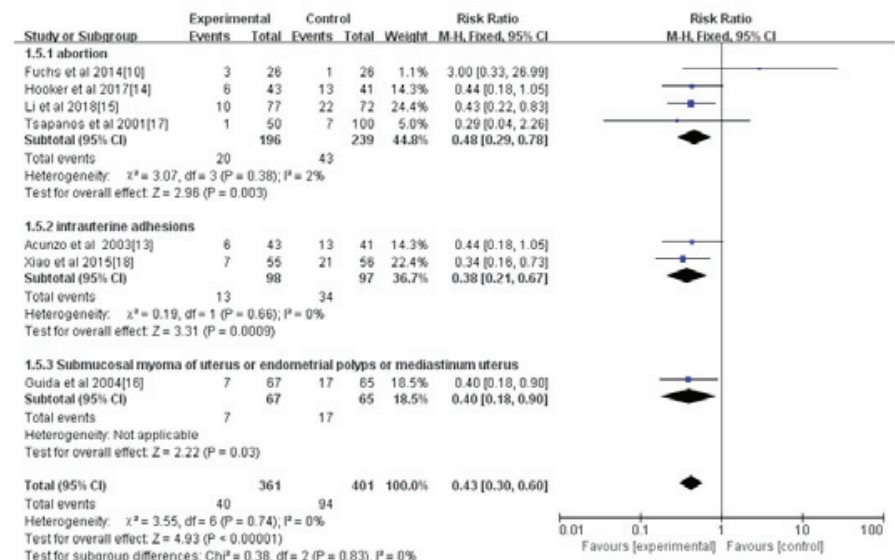

Figure 6. Forest plot of the incidence of postoperative intrauterine adhesion of different primary diseases after an intrauterine operation. CI, confidence interval; df, degrees of freedom; M-H, Mantel-Haenszel.

\begin{tabular}{|c|c|c|c|c|c|c|c|c|c|}
\hline Studv or Subgroup & \multicolumn{2}{|c|}{ Experimental } & \multicolumn{2}{|c|}{ Control } & \multicolumn{2}{|c|}{ Risk Ratio } & \multicolumn{3}{|c|}{$\begin{array}{c}\text { Risk Ratio } \\
\text { M-H, Fixed, 95\% Cl }\end{array}$} \\
\hline Fuchs et al 2014[10] & 7 & 26 & 3 & 26 & $10.1 \%$ & 2331068.8051 & & & \\
\hline Tsapanos et al $2001[17]$ & 38 & 50 & 40 & 100 & $89.9 \%$ & $1.90[1.43,2.53]$ & & & \\
\hline Total $(95 \% \mathrm{Cl})$ & & 76 & & 126 & $100.0 \%$ & $1.94[1.46,2.60]$ & & 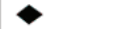 & \\
\hline Total events & 45 & & 43 & & & & & & \\
\hline $\begin{array}{l}\text { Heterogeneity. } \quad x^{2}=0.11 \\
\text { Test for overall effect: } Z=\end{array}$ & $\begin{array}{l}d f=1(P= \\
.51(P<0\end{array}$ & 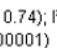 & $=0 \%$ & & & & $0.01 \quad 0.1$ & 10 & 100 \\
\hline
\end{tabular}

Figure 7. Forest plot of pregnancy rate after an intrauterine operation. CI, confidence interval; df, degrees of freedom; M-H, Mantel-Haenszel. 
by the type of intrauterine operation or the presence of primary diseases. The results of the current study also demonstrated that hyaluronic acid gels can significantly improve pregnancy rates following intrauterine operation. The seven studies included in the present analysis were similar in experimental design with no heterogeneity. In addition, the sample sizes were small and the follow-up times were short. These limitations may have affected the overall analysis. Therefore, a larger sample size with longer follow-up and appropriately designed randomized, controlled clinical studies are necessary to fully assess the efficacy of hyaluronic acid gel-assisted prevention of IUA following intrauterine operation. The results of the present study provide novel insight that may be used for the prevention of IUA.

\section{Acknowledgements}

The authors would like to thank Dr Zhu Bin and Dr Wu Zhengqiang in the Department of Obstetrics and Gynecology, Yiwu Maternity and Children Health Care Hospital (Jinhua, China) for their assistance in this study.

\section{Funding}

No funding was received.

\section{Availability of data and materials}

The datasets used and/or analyzed during the present study are available from the corresponding author on reasonable request.

\section{Authors' contributions}

FZ and XX contributed equally to this work by designing the study, reviewing literature, interpreting the analyses and writing the manuscript. FH and JL contributed to the literature review, data collection and analysis, and writing the manuscript. YC contributed to designing the study, data analysis, and writing the manuscript.

\section{Ethics approval and consent to participate}

Not applicable.

\section{Patient consent for publication}

Not applicable.

\section{Competing interests}

The authors declare that they have no competing interests.

\section{References}

1. Vancaillie TG and Garad R: Asherman's syndrome. Aust Nurs J 20: 34-36, 2013.

2. Yamamoto N, Takeuchi R, Izuchi D, Yuge N, Miyazaki M, Yasunaga M, Egashira K, Ueoka Y and Inoue Y: Hysteroscopic adhesiolysis for patients with Asherman's syndrome: Menstrual and fertility outcomes. Reprod Med Biol 12: 159-166, 2013.

3. Salazar CA, Isaacson K and Morris S: A comprehensive review of Asherman's syndrome. Curr Opin Obstet Gynecol 29: 249-256, 2017.
4. Mehedintu C, Ionescu O, Rotaru AM, Antonovici M, Plotogea M, Berceanu C, Vladareanu S and Bratila E: Asherman's syndrome after an uncomplicated cesarian section. Gineco 12: 166-168, 2016.

5. Friedman A, DeFazio J and DeCherney A: Severe obstetric complicantions after aggressive treatment of asherman syndrome. Obstet Gynecol 67: 864-867, 1986.

6. Bosteels J, Weyers S, D'Hooghe TM, Torrance H, Broekmans FJ, Chua SJ and Mol BWJ: Anti-adhesion therapy following operative hysteroscopy for treatment of female subfertility. Cochrane Database Syst Rev 11: CD011110, 2017.

7. Itano N: Simple primary structure, complex turnover regulation and multiple roles of hyaluronan. J Biochem 144: 131-137, 2008.

8. Salwowska NM, Bebenek KA, Żądło DA and Wcisło-Dziadecka DL: Physiochemical properties and application of hyaluronic acid: A systematic review. J Cosmet Dermatol 15: 520-526, 2016.

9. Hong MK and Ding DC: Seprafilm ${ }^{\circledR}$ application method in laparoscopic surgery. JSLS 21: e2016.00097, 2017.

10. Fuchs N, Smorgick N, Ben Ami I, Vaknin Z, Tovbin Y, Halperin R and Pansky M: Intercoat (Oxiplex/AP Gel) for preventing intrauterine adhesions after operative hysteroscopy for suspected retained products of conception: Double-blind, prospective, randomized pilot study. J Minim Invasive Gynecol 21: 126-130, 2014.

11. The American Fertility Society classifications of adnexal adhesions, distal tubal occlusion tubal occlusion secondary to tubal ligation, tubal pregnancies, Mullerian anomalies and intrauterine adhesions. Fertil Steril 49: 944-955, 1988.

12. Higgins JP and Green S: Cochrane handbook for systematic reviews of interventions. Cochrane Book Series, 2008.

13. Acunzo G, Guida M, Pellicano M, Tommaselli GA, Di Spiezio Sardo A, Bifulco G, Cirillo D, Taylor A and Nappi C: Effectiveness of auto-cross-linked hyaluronic acid gel in the prevention of intrauterine adhesions after hysteroscopic adhesiolysis: A prospective, randomized, controlled study. Hum Reprod 18: 1918-1921, 2003.

14. Hooker AB, de Leeuw R, van de Ven PM, Bakkum EA, Thurkow AL, Vogel NEA, van Vliet HAAM, Bongers MY, Emanuel MH, Verdonkschot AEM, et al: Prevalence of intrauterine adhesions after the application of hyaluronic acid gel after dilatation and curettage in women with at least one previous curettage: Short-term outcomes of a multicenter, prospective randomized controlled trial. Fertil Steril 107: 1223-1231.e3, 2017.

15. Li X, Wu L, Zhou Y, Fan X, Huang J, Wu J, Yu R, Lou J, Yang M, Yao Z and Xue M: New crosslinked hyaluronan gel for the prevention of intrauterine adhesions after dilation and curettage in patients with delayed miscarriage: A prospective, multicenter, randomized, controlled trial. J Minim Invasive Gynecol 26: 94-99, 2019.

16. Guida M, Acunzo G, Di Spiezio Sardo A, Bifulco G, Piccoli R, Pellicano M, Cerrota G, Cirillo D and Nappi C: Effectiveness of auto-crosslinked hyaluronic acid gel in the prevention of intrauterine adhesions after hysteroscopic surgery: A prospective, randomized, controlled study. Hum Reprod 19: 1461-1464, 2004.

17. Tsapanos VS, Stathopoulou LP, Papathanassopoulou VS and Tzingounis VA: The role of Seprafilm bioresorbable membrane in the prevention and therapy of endometrial synechiae. J Biomed Mater Res 63: 10-14, 2002.

18. Xiao S, Wan Y, Zou F, Ye M, Deng H, Ma J, Wei Y, Tan $\mathrm{C}$ and Xue M: Prevention of intrauterine adhesion with auto-crosslinked hyaluronic acid gel: A prospective, randomized, controlled clinical study. Zhonghua Fu Chan Ke Za Zhi 50: 32-36, 2015 (In Chinese).

19. Wang M, Liu C and Xiao W: Intra-articular injection of hyaluronic acid for the reduction in joint adhesion formation in a rabbit model of knee injury. Knee Surg Sports Traumatol Arthrosc 22: 1536-1540, 2013.

20. Knopf-Marques H, Pravda M, Wolfova L, Velebny V, Schaaf P, Vrana NE and Lavalle P: Hyaluronic acid and its derivatives in coating and delivery systems: Applications in tissue engineering, regenerative medicine and immunomodulation. Adv Healthc Mater 5: 2841-2855, 2016.

21. Huberlant S, Fernandez H, Vieille P, Khrouf M, Ulrich D, deTayrac R and Letouzey V: Application of a hyaluronic acid gel after intrauterine surgery may improve spontaneous fertility: A randomized controlled trial in New Zealand white rabbits. PLoS One 10: e0125610, 2015.

22. Khan Z and Goldberg JM: Hysteroscopic management of Asherman's syndrome. J Minim Invasive Gynecol 25: 218-228, 2018. 
23. Zhou Q, Wu X, Dai X, Yuan R and Qi H: The different dosages of estrogen affect endometrial fibrosis and receptivity, but not SDF-1/CXCR4 axis in the treatment of intrauterine adhesions. Gynecol Endocrinol 34: 49-55, 2017.

24. Xu W, Zhang Y, Yang Y, Zhang S and Lin X: Effect of early second-look hysteroscopy on reproductive outcomes after hysteroscopic adhesiolysis in patients with intrauterine adhesion, a retrospective study in China. Int J Surg 50: 49-54, 2018.

25. Cai H, Qiao L, Song K and He Y: Oxidized, regenerated cellulose adhesion barrier plus intrauterine device prevents recurrence after adhesiolysis for moderate to Severe intrauterine adhesions. J Minim Invasive Gynecol 24: 80-88, 2017.

26. Wei ML, Lin XN, Zhou F, Huang D, Kuang L and Zhang SY: Evaluation of safety and patient acceptance of uterine balloon stents in preventing intrauterine adhesions. Natl Med J China 94 3095-3097, 2014

27. Abu Rafea BF, Vilos GA, Oraif AM, Power SG, Cains JH and Vilos AG: Fertility and pregnancy outcomes following resectoscopic septum division with and without intrauterine balloon stenting: A randomized pilot study. Ann Saudi Med 33: 34-39, 2013.
28. Gupta S, Talaulikar VS, Onwude J and Manyonda I: A pilot study of Foley's catheter balloon for prevention of intrauterine adhesions following breach of uterine cavity in complex myoma surgery. Arch Gynecol Obstet 288: 829-832, 2013.

29. Krajčovičová R, Hudečk R, Ventruba $P$ and Surgentová K: The role of hyaluronan in Asherman's syndrome therapy. J Gynecol Surg 31: 250-254, 2015.

30. Back JH, Cho WJ, Kim JH, Park IK and Kwon SW: Application of hyaluronic acid/sodium alginate-based microparticles to prevent tissue adhesion in a rabbit model. Surg Today 46: 501-508, 2015.

(i) $\ominus$ This work is licensed under a Creative Commons Attribution-NonCommercial-NoDerivatives 4.0 International (CC BY-NC-ND 4.0) License. 